\title{
La acción humana: una propuesta integral para el diálogo interdisciplinar sobre la personalidad
}

\author{
Human Action: a Comprehensive Proposal \\ for Interdisciplinary Dialogue on Personality
}

\section{JOSÉ VÍCTOR ORÓN SEMPER}

Fundacion UpToYou Education josevictororon@gmail.com

ORCID: 0000-0003-4959-050X

\section{MIRIAM MARTÍNEZ MARES}

Fundacion UpToYou Education miriammartinezmares@gmail.com

ORCID: 0000-0003-4013-0769

Resumen: La necesidad de categorizar la personalidad es recurrente en estudios filosóficos, teológicos y, especialmente, psicológicos. No obstante, la falta de diálogo entre las distintas disciplinas da lugar a análisis que parecen dividir la realidad humana en 'partes', a menudo incompatibles entre sí. Atendiendo a este problema, el presente artículo propone el análisis integral de la acción humana (el modo en el que la persona se actualiza) buscando a una parametrización psicológica más ajustada a la complejidad de la personalidad. Partiendo de la visión integral de la acción, el aspecto comportamental y mental humanos comúnmente estudiados, se advierten como insuficientes y se amplía el horizonte con la referencia a la interioridad. Se pretende alcanzar una visión más acertada de la complejidad de la acción humana. La interioridad, parametrizada como identidad personal, intención relacional y posi- 
cionamiento existencial, reclama una lectura renovada y relacionada de la psicología, la filosofía y la teología.

Palabras clave: persona; actualización; comportamiento; mente; interioridad.

Abstract: The need to categorize the personality is recurrent in philosophical, theological and, especially, psychological studies. However, the lack of dialogue between the different disciplines gives rise to analyzes that seem to divide human reality into 'parts', often incompatible with each other. In view of this problem, this article proposes the integral analysis of human action (the way in which the person is actualized), seeking a psychological parameterization that is more adjusted to the complexity of the personality. Starting from the integral vision of the action, the human behavioral and mental aspects commonly studied are seen as insufficient and the horizon is broadened with the reference to interiority. It is intended to achieve a more accurate vision of the complexity of human action. Interiority, parameterized as personal identity, relational intention, and existential positioning, calls for a renewed and related understanding of psychology, philosophy, and theology.

Keywords: person; actualization; behavior; mind; interiority.

\section{Introducción}

El diálogo interdisciplinar es un requisito intrínseco al mismo conocimiento ya que ningún sistema se explica a sí mismo, sino que asume principios que no pertenecen a la propia disciplina o sistema de estudio (Orón Semper y Sánchez-Cañizares 2017). Esto se evidencia en el tema que nos concierne, a saber: el problema sobre la personalidad en el intento de responder a cómo se puede caracterizar la actualidad de una persona.

La referencia central en los estudios dedicados a la personalidad se orientan a la búsqueda de patrones de comportamiento, cognición y emoción (Corr y Matthews, 2009) pero se detecta en las distintas disciplinas una reducción de la persona a ciertas características de orden más bien mental que real. Siendo la conceptualización y categorización de la realidad la tarea imprescindible de toda ciencia en su ejercicio de comprensión y análisis de su objeto de estudio, ocurre que este mismo ejercicio de objetivación, en ocasiones y paradójicamente, puede perder de vista la especificidad de la realidad estudiada. Es la crítica que lanzan algunos filósofos 
contemporáneos, como Habermas (1990), al cientificismo1. El filósofo alemán critica el procedimentalismo propio de esa mentalidad que pretende garantizar el conocimiento cierto a precio de reducir lo real, únicamente, a todo aquello que sea determinable y abarcable por la razón. Pero este método deja de lado la experiencia para limitarse al experimento marginando, así, del ámbito cognoscitivo todo aquello que no se puede pesar ni medir.

Los reduccionismos propios de la mentalidad cientificista contemporánea afectan en el estudio de la persona y generan, así mismo, una apreciable dificultad de armonizar las diferentes aproximaciones a la personalidad. El presente trabajo propone, pues, el acto personal como el fenómeno que mejor revela la actualidad ${ }^{2}$ de la personalidad y que mejor responde tanto a la dificultad como a la necesidad de caracterizarla. Desde la perspectiva del acto humano, la parametrización de la personalidad comúnmente tratada, a saber, conductual y neurobiológicamente, se entiende como incompleta y se defiende, por lo tanto, incluir la dimensión de la 'interioridad' como una forma de hacer justicia a la realidad global de la persona.

Tanto el comportamiento como el funcionamiento del sistema nervioso han sido objeto de estudio para la psicología, la neurociencia y otras disciplinas que, a partir de sus análisis, han intentado aportar teorías sobre el ser humano y su naturaleza. Pero ninguno de estos dos elementos puede dar lugar a interpretaciones acertadas si no se analizan desde el fundamento que precede, atraviesa y explica la complejidad tanto de lo visible como de lo no visible de las acciones humanas, a saber, la interioridad. Tiene sentido afirmar que no es un mecanismo conductual el que ejecuta acciones, ni un cerebro el que piensa, sino que es ‘alguien’ quien actúa y ‘alguien’ el que piensa a través del cerebro... La visión integral de la acción supone atender la interioridad y, por lo tanto, la complejidad de elementos (también de orden existencial) que se ponen en juego en cada acción humana que se realiza. Proponemos que el acto humano no puede recoger

Actitud intelectual propiamente "positivista" muy influyente en la cultura contemporánea. Se entiende como "verdadero", exclusivamente, lo que la ciencia determina. El método científico-experimental adquiere, así, la función de filtrar los conocimientos que pueden considerarse válidos.

2 Se entiende por actualidad a la forma concreta y coyuntural sobre cómo una persona se constituye y manifiesta. 
toda su complejidad sin apelar a la interioridad que se reconoce en toda la actividad psíquica humana pero no es agotada por esta.

La tesis del presente artículo es una propuesta de la acción humana como manifestación de la condición interior de la persona y que precisa, para un análisis adecuado de la personalidad, la integración de la psicología, la filosofía y la teología. Referirnos a la actualidad desde esta perspectiva integral puede ser de gran utilidad para solventar la discrepancia que hay cuando se trata de indicar cuáles son los rasgos característicos de la personalidad; supondría abrir una posible vía ante la evidente dificultad en el ejercicio de su parametrización (Dubois, Eberhardt, Paul, y Adolphs, 2020; Goldberg y Saucier, 1998; Paunonen y Jackson, 2000).

\section{El acto humano, lugar de encuentro de la filosofía, la teología y la psicología en el estudio de la personalidad}

¿Existe una comprensión del actuar humano suficientemente abierta a la par que operativa para que pueda ser útil para los estudios interdisciplinares de la personalidad? A continuación, tratamos una perspectiva que, creemos, puede ser de ayuda en la filosofía, la psicología y la teología.

\subsection{La acción humana, ¿hacer o ser?}

El psicoanálisis revela que la persona responde en sus primeros años de vida de forma simultánea a las preguntas de ¿quién soy?, ¿quiénes son los demás?, ¿qué es el mundo? (Kohut, 2009; Orón Semper, Coello de Portugal, y Luis, 2020; Winnicott, 1986). La influencia entre las experiencias de la vida y las propias conceptualizaciones es bidireccional (Orón Semper, 2018b) de tal forma que, aun usando el mismo término, persona, nos referimos a realidades distintas, "únicas en su especie" (Wojtyla, 1982).

Las distintas experiencias de vida hacen que una persona se sienta afectada por la realidad de forma distinta y esta distinta afectación lleva a que se experimenten emociones que se expresan de muy diversos modos y genera un determinado tipo de actitudes que se reflejan en creencias: "Nuestras percepciones de las motivaciones propias (...) están a menudo 
limitadas por la forma de nuestra experiencia. (...) (Insensibles, personas fanáticas, alguien que no puede ver ciertas cosas). (...) La responsabilidad en este caso puede ser muy atenuada" (G. Taylor, 1985: 38-39). De ahí que cobre importancia facilitar modelos educativos aplicables desde los primeros años de vida y centrados en conseguir unas disposiciones interiores y no tanto comportamientos concretos (Orón Semper, 2020b). Dichos modelos se prolongan a lo largo de toda la vida bajo la forma de acompañamiento, surge así modelos donde la confianza se deposita en el educando como "persona que busca, que tiene la capacidad de conocer la realidad, de encontrarse con ella, recuperando el valor de la experiencia como campo de juego en el que la persona puede implicarse y desplegar todas sus facultades y dimensiones." (Gozález-Iglesias \& De la Calle Maldonado, 2020, 192).

La persona vive en estado permanente de proyección sobre la realidad en sus expectativas, creencias, deseos e ilusiones que han ido configurándose por la forma de dar significado a las diversas experiencias en un proceso muy subjetivo y relacional donde cobran vida las respuestas existenciales adoptadas. Las expectativas se verán confirmadas o negadas en el encuentro con la realidad y es en esa comunicación con lo real donde acontece la actualización de la persona. En ese sentido, podemos entender la personalidad como la caracterización de la actualización de la persona en cada uno de sus actos.

El término actualización está tomado del pensamiento de Whitehead y su propuesta de las entidades actuales, en las que converge el pasado, el presente y el futuro de la entidad (Whitehead, 1978). La actualidad es la forma concreta como la entidad se encuentra en un momento concreto. Que la actualidad revele presente, pasado y futuro en la coyuntura de un tiempo concreto, permite unificar lo manifestado y lo manifiesto de la persona sin desgajarla de su línea histórica vivida y por vivir. Se apela, aquí, a la acción humana como praxis y no sólo como poiesis o techné (Aristóteles, 2014).

Hay aspectos de la conducta del ser humano que pueden ser estudiados y los procesos mentales explican el porqué de muchos factores, pero una categorización limitada a lo comportamental y a lo mental deja de ser suficiente para explicar acciones capaces de proveer significados, de te- 
ner un lenguaje simbólico, de evocar lo inmaterial y proyectar, en las acciones, un sentido que va más allá del puro bienestar físico y psicológico. Así pues, entender la personalidad en relación con el actuar humano (como praxis) parece que puede resultar más provechoso, dado que pasamos del reducido escenario del hacer y sus mecanismos al horizonte del ser y su misterio.

\subsection{La triple dimensión del actuar humano}

La presente defensa se inspira en la teoría de los tres radicales de Leonardo Polo; se trata de una propuesta filosófica (Polo, 2007b: 181-242) que se ha mostrado muy útil en teología (Orón Semper, 2018c) en psicología (Orón Semper, 2019b) y en educación (Orón Semper, 2018a, 2020a). Tomando nota de lo ya publicado, se puede expresar brevemente de la siguiente manera.

Polo habla en concreto de tres radicales ${ }^{3}$ haciendo un gran resumen de la historia de la filosofía: a) el radical clásico resalta que se actúa desde la esencia humana perfeccionada con hábitos y virtudes; b) el radical moderno (en referencia al modernismo y postmodernismo), resalta el carácter productivo del actuar, por el que se introduce novedad en la historia; y c) el radical cristiano (en referencia al desarrollo medieval y posterior) resalta que, en su actuar, la persona crece también; en otras palabras, el ser no solo precede sino que también sigue al obrar. Estos tres radicales podrían reformularse, respectivamente, como el radical de la naturaleza humana, el radical del sujeto y el radical personal. Se trata de una división, ciertamente, artificiosa que, además, no apunta a cuestiones contradictorias de la naturaleza humana. Sin embargo, se trae a colación ya que cumple una función orientativa en la presente interpretación de la acción humana.

A nuestro parecer, es preciso integrar la visión antropológica tanto de los clásicos como de los modernos (en sus vertientes más objetivistas y subjetivistas, respectivamente) en el "radical personal". De este modo, se parte de la novedad en la que consiste cada persona cuya realidad sub-

3 El término 'radical' hay que entenderlo en el sentido de raíz, de tal forma que lo radical muestra lo que se enraíza/echa raíces en el ser humano y su actuar. 
jetiva apunta a algo más allá de su ser 'sujeto', es decir, de su estado de conciencia subjetivo. J. Crosby habla de autopresencia ${ }^{4}$ y K. Wojtyla de autodeterminación ${ }^{5}$ para apuntar a la acción personal como el desarrollo del 'ser' que le es dado y desde su propia libertad. De este modo, se afirma que

El radical clásico o de la naturaleza humana descubre que hay una realidad que el ser humano recibe, que es su naturaleza, y evidencia que la verdad no es creación humana, pues lo verdadero es estable. También advierte que hay algo inmaterial en el ser humano que supera el tiempo, y que al poner en acto sus facultades, estas mejoran. El ser humano es acto en sus actos. El radical moderno o del sujeto es productivo y aporta la novedad cultural. Pero 'producir' no es 'causar', porque el efecto de la causa no comporta novedad. El radical cristiano o personal señala que se está llamado a trascenderse, a encontrarse con las demás personas creadas e increadas. Hay en él, por tanto, una libertad de destinación. (Orón Semper, 2018c).

Inspirados, pues, en la distinción de Leonardo Polo, podemos formular que la acción humana tiene tres grandes dimensiones: la comportamental, la mental y la interioridad, de modo que la clave de interpretación que asumimos es la libertad o intimidad desde la que el ser humano vive y se vive a sí mismo corporalmente. Mirar hacia lo irreductible del ser humano es la condición para verlo en su esencia, esto es, en su condición de persona; "es necesario detenerse en lo que es irreductible en lo que en todo hombre es único y no repetible, aquello por lo que cada hombre es no sólo 'hombre-individuo' de un género [...] es persona-sujeto.” (Wojtyla,1987: 34). Las dimensiones comportamentales y mentales son las más fáciles de identificar. Lo mental o psíquico está referido a la inteligencia, la voluntad, las emociones, la imaginación, lo sensorial, etcétera. El término interioridad remite a la intimidad que acontece en cada uno de nosotros.

4 Término que utiliza Crosby para referirse a esa cierta independencia de la persona en su ser y en su obrar, a su ser "radicalmente suyo" con una "plenitud particular de identidad y de ser incomunicable.” (2007: 69).

5 Término muy recurrente en K. Wojtyla (1982) para explicar la forma propia en la que acontece la acción humana dada la conciencia personal: además de su función intencional (“de reflejo”) su función reflexiva da lugar a la autoposesión. 
Esta propuesta bebe de las raíces agustinianas y se aleja del intelectualismo en el que se entiende al ser humano, esencialmente, como un ser racional. San Agustín, en cambio, habla desde una perspectiva integral, expresándolo como un ser relacional, esto es, un ser de encuentro. San Agustín entiende, pues, la intimidad como un lugar de encuentro: "Porque tú estabas dentro de mí, más interior que lo más íntimo mío y más elevado que lo más sumo mío" (Confesiones, III, 6, 11). El 'corazón' del lenguaje agustiniano trae a la persona completa, desde el centro mismo de su ser, a la relación con lo real. La intimidad no es una relación cognitiva y alejada, sino una relación personal amorosa (Flamarique, 2016) que se aplica a todo encuentro entre personas.

De este modo, no se entiende el 'pensar' como un ejercicio especulativo desde el que se objetiva la realidad. Todas las propuestas personalistas parten de esta ampliación fenomenológica del conocimiento a la vida afectiva de la persona, entendiendo que ésta queda vinculada a la realidad desde la interioridad, desde la unidad de su ser. Por eso dirá Wojtyla que la realidad se habita como 'mundo' indicando que "se incorpora definitivamente a la esfera de la subjetividad individual de cada ego humano." (1982: 53).

Hablar de intimidad es hablar de una realidad transcendente que no permite ser agotada en ninguna caracterización. A este respecto, Levinas explica que la alteridad de la realidad personal trasciende al sujeto de modo que, a diferencia del idealismo kantiano, no se interpreta la condición del 'Otro' a fenómeno de conciencia. (Pérez, 2008: 70)6 .

Se defiende, pues, que la experiencia integral de la acción personal hace posible y deseable el encuentro interdisciplinar entre la psicología, la filosofía y la teología. Abrir el escenario de comprensión de la personalidad a su habitar el mundo íntimamente (como relación y no sólo desde la razón), a nuestro parecer, permite acoger los muchos aspectos de la personalidad e impide su reducción a tareas específicas, a comportamientos tipo o a mecanismos de reacción físico-químicas del sistema nervioso.

Diversos autores, de la corriente fenomenológico-realista y personalista, han apostado por recalcar este peculiar modo de ser trascendente propio de la condición libre del ser humano, como Robert Speamann (2000), Leonardo Polo (1998, 2007a), Emmanuel Levinas (1993), etc. 


\section{Comportamiento-mente-interioridad, una propuesta de parametrización de la personalidad}

Nuestra propuesta surge de la inquietud sobre cómo parametrizar la actualización de la personalidad sin caer en reduccionismos y con la intención de hacer la mayor justicia posible de la realidad total del ser humano. Incluir la interioridad en la caracterización de su acción supondría ampliar la perspectiva y necesitar, así, del diálogo interdisciplinar entre psicología, filosofía y teología.

Se quiere hacer una propuesta que de sustento a la unidad de la acción humana. No solo se quiere afirmar la unidad de las potencias humanas que ya fue presentado con mucha consistencia por Tomás de Aquino (Enrique Gómez \& Montoya Camacho 2021), sino de una unidad superior que es la persona hecha presente desde su intimidad, lo cual explica la unidad de las potencias humanas.

Podríamos presentar la complejidad advertida en la persona desde el siguiente esquema:

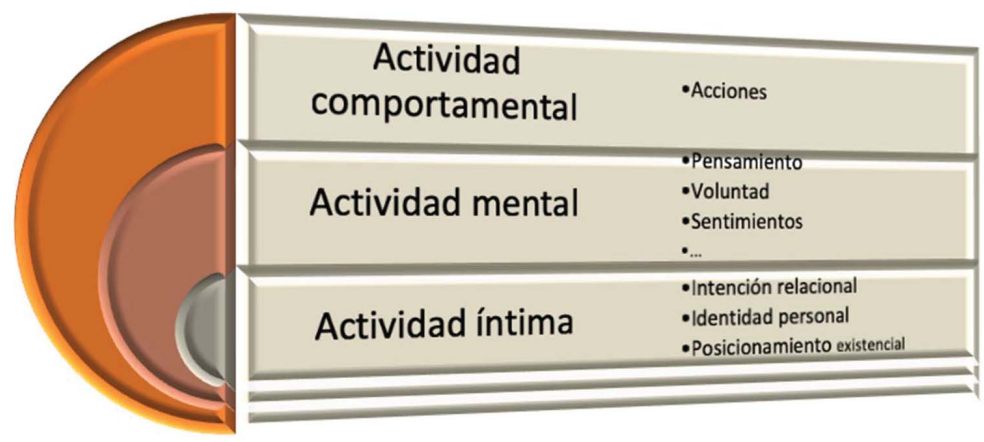

'Estructurar' la realidad personal no es otra cosa que una presentación psicológica de algo que supera el campo de la psicología. La persona es persona, precisamente porque es intimidad; es decir, la persona es más que cualquier caracterización que se haga de la misma. Es más fácil hablar de la interioridad por lo que no es que por lo que es. Cualquier caracteri- 
zación de la persona es insuficiente, no por desconocimiento, sino porque hay algo que se resiste a ser caracterizado en una explicación cerrada. Es por eso que se trata de una presentación psicológica de algo no psicológico. El esquema quiere representar la acción humana y no quién es la persona; es decir, no es un esquema antropológico.

La actividad comportamental, la mental y a la intimidad son expresadas como 'dimensiones' para evitar que puedan entenderse como realidades independientes. Dimensionar es algo que hacemos cognitivamente. De la misma forma ocurre con un objeto, que puede ser 'dividido' en sus proyecciones por los ejes de coordenadas en planta, alzado y perfil, pero se trata siempre del mismo objeto.

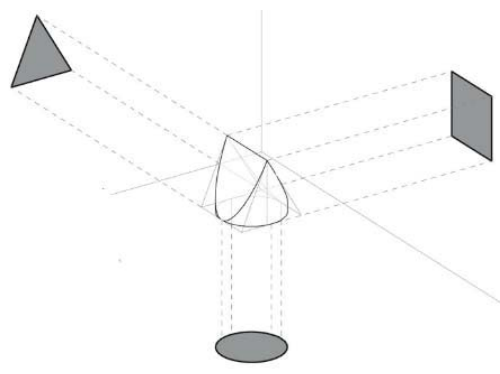

La dimensión comportamental, mental e interioridad surge de hacerse preguntas diversas sobre la misma acción humana. Esa sería la forma de "proyectar sobre los ejes" la acción humana. Preguntarse es focalizarse en ciertas realidades de interés y al hacer eso sesgamos cognitivamente la realidad. Cuando nos preguntamos sobre qué aspectos tiene el acto humano que pudieran ser medidos con una regla y un reloj, estamos hablando del comportamiento. Cuando nos preguntamos sobre qué aspectos tiene el acto humano que la persona puede reportar, aunque no se ven, hablamos de lo mental y la intimidad; respecto a aquellos que están relacionados a cómo la persona actúa sobre el mundo, nos referimos a lo mental y cuando nos preguntamos cuáles de ellos están ligados a todo un sistema de creencias desde los que la persona actúa sobre sí misma y sus relaciones, surge la interioridad. 
La división de la acción humana en su dimensión comportamental, mental o interior es, pues, una artificialidad: ninguna puede existir por separado. Incluso un gesto tan sencillo como levantar la mano para saludar a alguien supone tener una intención relacional, una respuesta a la pregunta de quién soy, un posicionamiento existencial, una actividad intelectual, volitiva, memorística, emocional, imaginativa, etcétera y un movimiento corporal. Sin todas ellas, la mano no se mueve, a no ser que se trate de un arte reflejo. Así pues, la tríada comportamiento-mente-intimidad es el resultado de focalizaciones conceptuales respecto al acto humano y no pretende reflejar, como se ha dicho, una realidad "dimensionada" del ser humano.

\subsection{Parametrización de la interioridad}

Al hablar de la acción humana, no parece necesario detallar las dimensiones comportamental y mental, pero sí es necesario explicar por qué se relaciona la intimidad. Pues bien, la intimidad despierta la cuestión fundamental sobre el dinamismo propio del acto humano en tres aspectos, a saber: el posicionamiento existencial, la intención relacional y la identidad personal. Estos tres términos no se refieren a ningún objeto en concreto ni hacen referencia a ninguna coyuntura, sino a tres disposiciones generales de la persona que, existencialmente, se expresa como un mismo dinamismo, conforman un mismo sistema de creencias que bebe de la experiencia; la explicación exhaustiva de uno lleva a ir mencionando a los demás, pues mutuamente se reclaman. Dicha unificación es evidenciada por la teoría del apego (Bowlby, 1982; Pittman, Keiley, Kerpelman, y Vaughn, 2011) y la referencia social (Dickstein y Parke, 1988; Walden y Kim, 2005; Walden y Ogan, 1988).

El posicionamiento existencial se refiere a una disposición basal de la persona hacia la vida en general. Se ha hecho referencia a este hecho desde varios ámbitos. Por ejemplo desde el campo de la ética con una lectura fenomenológica a través del término "disposición basal de fondo" (Crespo, 2009), o desde la teología moral con el término "opción fundamental” (Herráez, 1978; Nello, 1995), o desde la psicología como el "sentido de vida" (Frankl, 1984, 2000). 
La identidad personal es la respuesta que cada uno da a la pregunta “quién soy yo?”. La pregunta no es ¿quién es el hombre?, sino quién es uno mismo. Un gran desarrollo de los temas identitarios lo encontramos en Erikson $(1959,1968)$ desde la psicología y en Taylor (C. Taylor, 2006) desde la filosofía. Es reflejada como una realidad subjetiva y un tema recurrente en la vida de la persona que emerge cuando la persona descubre que el futuro no tiene por qué ser igual que el pasado y que uno, en el desarrollo de su autoría, no solo transforma el mundo, sino que se transforma a sí mismo.

La intención relacional se trata de la disposición de la persona ante las otras personas que nos constituye en quienes somos (Buber, 1982; Levinas, 1993) y que, básicamente, puede concretarse en una disposición a la confianza o, por el contrario, a la necesidad de asegurar (Erikson, 1963 configuración realizada en la época de 0 a 2 años). Estas dos disposiciones entienden la relación yo - tú - mundo de forma distinta. La confianza usa el mundo para ponerlo al servicio del encuentro interpersonal mientras que la seguridad usa las relaciones interpersonales para dominar el mundo (Orón Semper, 2020b). Otra forma similar de entender la intención relacional es a través de los estudios antropológicos que señalan que las personas desarrollan una intención compartida basada en la confianza mientras que los animales desarrollan una intención individual similar basada en asegurar la supervivencia del individuo (Tomasello, 2007, 2014).

\subsection{La interioridad, límite en la parametrización de personalidad}

Al hablar de interioridad en relación con la identidad, la intención relacional y el posicionamiento existencial, no se dice nada sobre qué es la interioridad o la intimidad, sino sobre cuál es la vía de actualización en la acción humana, sobre lo que supone ser intimidad. En otras palabras, al hablar de intención, de identidad personal y de posicionamiento existencial, a lo que se alude es a la repercusión de la interioridad en el actuar.

Hablar de la interioridad es afirmar que la persona es libertad (Polo, 1998) y que la libertad humana supera la causalidad mecánica que se descubre por la experiencia (Polo, 1997: capítulo V). Desde esta perspectiva, la ecuación conductista 'estímulo-respuesta' no resuelve el problema. El 
análisis del funcionamiento del cerebro lleva a Arolas a la siguiente afirmación: "La neurona es fiel. De esto se deduce que el depósito o sistema referencial que han dejado en la infancia nuestros padres y maestros será importante en el futuro" (2004: 128). Sin embargo, el impacto de las experiencias y las respuestas adquiridas y aprendidas a partir de éstas se enfrenta a la demostrada plasticidad del cerebro por la que una persona es capaz de desaprender ciertos hábitos adquiridos, reinterpretar sus experiencias o, incluso, abandonar adicciones. ¿Será que la persona no está sujeta a la mera interacción fisicoquímica de las neuronas?; si bien no es posible ejecutar ninguna acción sin servirse de procesos bioquímicos y mentales, también parece lógico afirmar que el ser humano "es más que la suma de estos procesos." (Arolas, 2004: 119).

Así pues, lo que aquí se presenta es una acción humana condicionada pero no determinada y, por lo tanto, fundamentada en la libertad como elemento peculiar que hace del ser humano un ser abierto siempre a la novedad, un ser que es "más” que sus comportamientos (Polo, 1998), un ser que es "único en su especie” tal y como pretende expresar el término persona (Wojtyla, 1982).

El problema que se trae a colación se reconoce en el clásico dilema alma-cuerpo o mente-cerebro. Desde la perspectiva integral se propone la conciliación (o reconciliación) entre ambos; ni la interioridad margina el cerebro, ni el cerebro margina la interioridad. El sistema nervioso, en su estructura cerebral, apunta a la participación de la interioridad. La flexibilidad de la mente misma, en su estructura cerebral, permitiría la participación de la interioridad. Podríamos hablar de una biología abierta, una biología capaz es decir, con capacidad de acogida de la interioridad. Así podría ocurrir que lo que sostiene todo el proceso de desarrollo de la proyección, la identidad y la relación es un “yo" intransferible e incomunicable. 


\section{La interioridad, revolución para las distintas disciplinas en el estudio de la personalidad}

A continuación, tratamos la utilidad de la propuesta integral del actuar humano para diversos temas de personalidad en relación con la teología, la filosofía y la psicología. La visión integrada de la propuesta de la acción resuelve diversas problemáticas clásicas relacionadas entre sí: la unidad de la acción, la valoración ética de la acción, la experiencia moral y ciertas cuestiones de la personalidad como hasta qué punto es algo estable o cambiante en la persona.

De la mano de las herramientas conceptuales y hermenéuticas de las que dota la filosofía (al tratar sobre las facultades humanas, la libertad, la búsqueda de sentido, etc.), y de los presupuestos de carácter teológico (en lo concerniente a lo trascendente y espiritual del ser humano), la psicología trata de entender cómo la persona se actualiza y qué se puede saber de la persona por sus manifestaciones.

El psicoterapeuta se enfrenta cotidianamente con la existencia espiritual, en términos de libertad y responsabilidad, y enfrentándolas a la facticidad psicofísica sólo consigue que el paciente se entregue a su destino fatal. Contra este fatalismo neurótico sólo cabe tomar conciencia de la libertad y la responsabilidad. (Frankl, 1977:14).

Pero los intereses son bidireccionales; en la medida en que se requiere correspondencia entre la forma de comprender a la persona y su manifestación, la teología y la filosofía beben de los descubrimientos psicológicos y sus estudios de índole experimental que insertan la experiencia humana en la corporalidad psíquica y emocional de la persona, constituyendo el hecho empírico que analizan estas ciencias. Para la filosofía, aportaciones psicológicas como, por ejemplo, los procesos de la actividad volitiva, son un instrumento importante de la reflexión ética sobre la libertad humana (Wojtyla, 1997: 153). En teología, por su parte, entender cómo se abre camino la gracia en la naturaleza psíquica de una persona es una cuestión 
fundamental para el acompañamiento pastoral y para toda la temática de carácter ético.

Partir de esta experiencia singular y concreta de la acción personal para el posterior análisis teórico permite que, en la comprensión de la personalidad, cobre protagonismo la persona como "sujeto-de su existir y de su obrar.” (Wojtyla, 1982: 90). De esta manera, en el ámbito práctico de la moralidad (y, consecuentemente, en el ámbito educativo) enunciar lo que 'debe' ser hecho con carácter universal y abstracto, carece de sentido. El moralismo cae en la rigidez de anteponer lo normativo (genérico y abstracto) a la situación concreta y así se confunde el 'necesitar' o 'tener' con el 'deber'; no es, por ejemplo, lo mismo necesitar dormir que deber dormir. El acto bueno necesita ser discernido y no hay forma de resolverlo a un nivel teórico. Se está apelando a la virtud de la prudencia que es una virtud eminentemente práctica (Aristóteles, 2014: libro sexto, capítulo IV) y que revela a la persona como causa de su acción, y no un simple medio de fines propuestos por una razón universal y, en esta medida, impersonal como si, “al perseguir cada cual su propósito, según su talante [...] siguieran insensiblemente, como hilo conductor, la intención de la naturaleza.” (Kant, 1994: 41).

Para la teología moral, acercarse a la concreción de la persona que se actualiza a sí misma en la acción, desde su centro libre, supone solventar la crítica a la propuesta de la opción fundamental donde parece que el acto se define simplemente por una disposición general en contraposición al comportamiento (Nello, 1995). La complejidad que se abre ante las distintas dimensiones del acto humano conduce al rechazado de cualquiera de los extremos; tanto aquel que dice que salvar la intención es suficiente para valorar como bueno el acto moral, así como aquel que defiende que existe un comportamiento correcto universalizable. Lo bueno no es posible conocerlo al margen de su realización concreta porque lo bueno, como sustantivo, no existe, sino que existe como calificativo de una acción personal (adverbio).

La caracterización de la acción humana guarda relación, así mismo, con el tratado teológico sobre la gracia y el pecado. ¿Cómo queda afectada la persona por el encuentro de gracia o por la vida aislada de pecado? Desvin- 
cular el comportamiento de la interioridad generaría una lectura incompleta de la experiencia religiosa: una simple relación del ser humano con ideales. No obstante, ¿dónde queda la fe como una genuina relación con la persona de Dios? (Orón Semper, 2018c). La desintegración del ser humano, cuando se obvia su interioridad, genera el moralismo, la ideología y el activismo que, según el pensamiento de Ratzinger (1990), hacen del cristianismo una realidad sin experiencia interior (Téllez Maqueo, 2013).

La actitud moralista pretende justificar un comportamiento apelando a un principio formulado de forma genérica, idealizada. Recuperar, pues, el escenario real en el que la persona se actualiza a sí misma permitirá abandonar los esfuerzos filosóficos y teológicos por definir qué es la persona desde un pensamiento abstracto y alejado de la experiencia. Tanto el moralismo como el activismo establecen una desconexión entre lo formulado y la vida real de forma que, por ejemplo, la persona que actúa puede limitarse a 'cumplir' unas normas, no siendo esto indicador de que el bien sea un valor querido y experimentado interiormente. La acción queda, así, desarraigada de la persona que actúa y su experiencia personal.

En el escenario de la educación, la idealización origina un trabajo de ‘tecnificación' del proceso educativo determinando objetivos y competencias a alcanzar como finalidad de la acción de educar. Se trata de una praxis humana resultista o utilitarista en la que la persona deja de ser fin de las acciones y parece un simple instrumento en nombre de ciertos objetivos morales y aspiraciones sociales. El educador se convierte en simple medio para que el educando alcance su objetivo (adquirir $x$ competencia) $y$, a su vez, el educando se convierte en medio al servicio de la competencia que se espera que adquiera. Tal relación interpersonal reducida al uso de la relación y de la persona para alcanzar determinados fines supone originar una educación despersonalizante o deshumanizante (Orón Semper, 2019a).

La racionalidad instrumental como modelo de actuación humana se expresa en las diferentes culturas de muy diversas formas, y es el tipo de praxis criticada desde una filosofía y teología de tinte fenomenológico propio de los personalismos, centrados en la persona desde su singularidad (Buber, 1982; Levinas, 1993; Polo, 1998; Spaemann, 2000) o en su 
crecimiento (Orón Semper, 2016); de modo que la educación se propondría, más bien, como un acompañamiento (González-Iglesias \& De la Calle, 2020) en el que "no se trata de impactar con dinámicas que atraigan superficialmente su atención. Se trata más bien de desvelar, sacar a la luz, de dentro afuera. Saber despertar sus preguntas existenciales y ponerlas en juego para, desde ellas, iluminar su camino de crecimiento." (190). De este modo, el acompañamiento se asume "como un camino de encuentros orientados a la plenitud de la persona" (181) en el que los objetivos morales o curriculares deseables para el educando apuntan, más bien, a su crecimiento en la medida en que se trabaja desde su interioridad.

La presentación de la acción humana desde una perspectiva integral pretende, pues, solventar estos problemas defendiendo la unidad de la acción humana, que es lo mismo que entender que la interioridad no puede desgajarse de la actividad mental y la comportamental. No obstante, si bien se propone la interioridad como elemento que hace falta implementar en el intento de caracterizar la actualización de la personalidad para respetar su globalidad, se advierte, a su vez, el límite de este intento, pues se precisa psicologizar la interioridad parametrizándola en intención relacional, posicionamiento existencial e identidad personal. Puestos a categorizar, más vale hacerlo desde la mayor integración posible, pero afirmar la interioridad es admitir su resistencia a ser parametrizada propiamente. De esta manera, queda también desmentida la idealización de la persona y cualquier ejercicio (ya sea psicológico, religioso, moral o educativo) que pretenda reducir la actualización de la persona a mecanismos meramente comportamentales y categorizables que obvien la experiencia subjetiva, íntima y singular de la persona en su acción.

Lo que en definitiva se defiende es la recuperación de la persona como causa de su acción y, en cierto modo, causa sui, esto es, principio y fin de ciertos comportamientos reflejados en la actividad mental que no apela a otra cosa que a la interioridad que experimenta el mundo desde su centro viviente y actualizante. Es por este motivo que se hace hincapié en que estamos tratando una propuesta de la acción humana y no respondiendo a quién es la persona y es también por esto que se hace preciso la comu- 
nicación entre la psicología, la filosofía y la teología. Al fin y al cabo, la interioridad no resuelve el problema, sino que muestra su complejidad.

\section{Conclusión}

El reconocimiento de la actividad interior y la caracterización psicológica de la interioridad supera los límites del idealismo y de la cultura moderna que acaba caracterizando a la persona por su actividad (Orón Semper, 2015), permitiendo caracterizaciones más abiertas (Akrivou y Orón Semper, 2016; Akrivou, Orón Semper, y Scalzo, 2018). Supone abrir el horizonte de comprensión de la personalidad a la dimensión interior que no agota a la persona. Parametrizar la dimensión interior en intención relacional, posicionamiento existencial e identidad personal como una lectura psicológica y no antropológica hace que la acción humana apunte a algo más que un sujeto mental al mismo tiempo que no agota al sujeto personal en su acción. Esto explicaría que pueda introducir novedad, fenómeno que no se explica ni por la biología, ni por la psique. Si se considera la acción humana de una forma suficientemente amplia, se podrá llegar a una visión del sujeto mucho más completa, no como mero actor de la acción sino como autor de una vida.

La presentación del acto humano aquí expuesta permite que el análisis de la personalidad se abra a la complejidad. Las tres dimensiones del acto humano no se relacionan de forma unívoca, es decir, la interioridad en acto no implica una activación mental y comportamental concreta. La referencia a la interioridad sirve tanto para dar la estabilidad más allá del comportamiento coyuntural del sujeto siendo la fuente unificadora del presente, pasado y futuro, como para dar cuenta de la libertad de la persona. Así, al actuar, su propia autodeterminación no la determina de una vez por todas, sino que sigue siendo siempre 'más' que sus acciones. Sin la interioridad como centro interpretativo de la personalidad, no habría un 'alguien' concreto que se actualiza, ni un verdadero 'actualizarse' a sí mismo, pues este 'sí mismo' no sería más que una secuencia de acciones separadas y desconectadas entre sí. 
La interioridad como referencia, ofrece parámetros caracterizables para tener una visión más amplia de la personalidad. En concreto en este artículo hemos propuesto la identidad personal, la intención relacional y el posición existencial. Sin la apelación a la interioridad, cualquier teoría de la acción humana quedaría incompleta. Atender la libertad del acto humano hace que toda caracterización sea caduca; las teorías de la personalidad tendrán siempre el límite de que la libertad humana no es caracterizable. Sin embargo, esta libertad parece ser el fundamento último y radical que explica la actualización de la persona en sus múltiples formas de vivir sus disposiciones y dinamizar sus dimensiones. Que la libertad esté detrás de este fenómeno permite presentar el esquema del acto humano con su triple dimensión comportamental-mental-interioridad y da cuenta de la realidad, en cierto sentido, incomunicable e inabarcable que es la persona.

\section{Bilbliografía}

Akrivou, Kleio, y Orón Semper, José Víctor. 2016. “Two kinds of human integrity: Towards the ethics of the inter-processual self (IPS).” En Challenges of Capitalism for the Common Good and Virtue Ethics, edited by Kleio Akrivou \& Alejo Sison, 221-253. Elgar.

Akrivou, Kleio, Orón Semper, José Víctor, y Scalzo, German. 2018. The Inter-Processual Self. Towards a Personalist Virtue Ethics Proposal for Human Agency. Cambridge: Cambridge Scholars Publishing.

Aristóteles. 2014. Ética a Nicómaco. Madrid: Gredos.

Bowlby, John. 1982. Attachment and loss. Vol 1. Attachment. (Second.). New York: Basic Books.

Buber, Martín. 1982. Yo y tu. Buenos Aires: Ediciones Nueva Visión.

Corr, Philip J., y Matthews, Gerald (Eds.). 2009. The Cambridge Handbook of Personality Psychology. Cambridge: Cambridge University Press.

Crespo, Mariano. 2009. “Sobre las disposiciones morales de fondo.” Thémata. Revista de Filosofía 41: 144-160.

de Aquino, Tomás. 1989. Suma de teología. Parte I-II (Segunda.). Madrid: Biblioteca de Autores Cristianos.

Crosby, John. 2007. La Interioridad de la Persona Humana. Hacia una antropología personalista. Madrid: Encuentro. 
Dickstein, S., y Parke, R. D. 1988. "Social referencing in infancy: a glance at fathers and marriage." Child Dev. 59 (2): 506-511.

Dubois, J., Eberhardt, F., Paul, L. K., y Adolphs, R. 2020. “Personality beyond taxonomy.” Nature human behaviour 4 (11): 1110-1117. doi:https://doi.org/10.1038/ s41562-020-00989-3

Enrique Gómez, M.T. y Montoya Camacho, J.M. 2021 “Imperium and causality in Thomas Aquinas.” Scientia et Fides, 9 (1): 329-355. DOI: http://dx.doi. org/10.12775/SetF.2021.013

Erikson, Erik H. 1959. “Identity and the cycle of life: selected papers.” Psychological issues 1 (1): 5-165.

Erikson, Erik H. 1963. Childhood and society (2nd ed.). New York: W.W: Norton.

Erikson, Erik H. 1968. Identity: youth and crisis. New York: W.W: Norton.

Flamarique, L. 2016. La fenomenología de la interioridad en Agustín de Hiponay su interpretación existencial en Kierkegaard y Heidegger. Anuario filosófico 49 (2): 317-338.

Frankl, Viktor E. 1984. El hombre en busca de sentido. Barcelona: Herder.

Frankl, Viktor E. 2000. En el principio era el sentido. Reflexiones en torno al ser humano. Barcelona: Paidos.

Frankl, Viktor E. 1977. La presencia ignorada de Dios. Psicoterapia y religión, Barcelona: Herder, Trad. J. M. López Castro.

Frisina, Warren G. 2002. The unity of knowledge and action. Towards a nonrepresentational theory of knowledge. New York: State University of New York Press.

Glas, Gerrit. 2006. "Person, Personality, Self, and Identity: A Philosophically Informed Conceptual Analysis.” Journal of personality disorders 20: 126-138; discussion 181. doi:10.1521/pedi.2006.20.2.126

Goldberg, Lewis R., y Saucier, Gerard. 1998. “What Is Beyond the Big Five?” Journal of Personality 66 (4), 495-524. DOI:https://doi.org/10.1111/1467-6494.00022

González-Iglesia, Sonia M., De la Calle Maldonado, Carmen. “El acompañamiento educativo, una mirada ampliada desde la antropología personalista." Scientia et Fides [S.1.] 8 (1): 183-203. DOI:http://dx.doi.org/10.12775/SetF.2020.012.

Habermas, J. 1990. Pensamiento postmetafísico. Madrid: Taurus.

Herráez, Fidel. 1978. La opción fundamental. Salamanca: Sigueme.

Kant, Immanuel. 1994. Idea de una historia universal en sentido cosmopolita, Madrid: Tecnos.

Kohut, Heinz. 2009. The restoration of the Self. Chicago - London: The University of Chicago Press.

Levinas, E. 1993. Humanismo del otro hombre (Colección.). Madrid: Caparrós editores. 
Nello, Antonio. 1995. Teorema de la opción fundamental. Bases para su adecuada utilización en la teología moral. Roma: Gregorian University Press.

Orón Semper, José Víctor. 2015. “Leonardo Polo’s integrative dynamic as a philosophical framework for understanding neuroscience. Journal of Polian Studies 2, 109-133.

Orón Semper, José Víctor. 2016. "Virtud como comportamiento ideal o como dinámica interior.” Estudios filosóficos Polianos 3, 22-28.

Orón Semper, José Víctor. 2018a. “Educación centrada en el crecimiento de la relación interpersonal. Studia Polaina 241-262.

Orón Semper, José Víctor. 2018b. “Emoción, significado, cosmovisión y autoconcepto, un arcoíris conceptual.

Orón Semper, José Víctor. 2018c. “Repercusiones teológicas de la dinámica de la integración.” Teología Espiritual, LXII, 63-163.

Orón Semper, José Víctor. 2019a. “Implicaciones de la relación entre el acto de ser y la esencia. En Juan A. García González (Ed.), Sobre la filosofía de Leonardo Polo, Familia, Educación y Economía (pp. 364-375). Madrid: Ideas y Libros Ediciones.

Orón Semper, José Víctor. 2019b. “Modelo antropológico subyacente a la propuesta dominante de la regulación emocional.” Quien 9, 9-38.

Orón Semper, José Víctor. 2020a. Encuentro Interprocesual. Para un mundo para el encuentro interpersonal. Madrid: ICCE.

Orón Semper, José Víctor. 2020b. “UpToYou, emotional education for personal growth in the early years. Educating interior dispositions. En People, Care and Work in the Home (p. in press). London: Routledge.

Orón Semper, José Víctor, Coello de Portugal, Sonsoles Navarro-Rubio, y Luis, Elkin. 2020. "Emotional education for personal growth in the early years." Journal of Theoretical and Philosophical Psychology. doi:10.1037/teo0000150

Orón Semper, José Víctor, y Sánchez-Cañizares, Javier. 2017. “Es posible la reducción epistemológica? Todo sistema necesita presupuestos extra-sistémicos.” Anuario filosófico 50 (3), 601-617. doi:10.15581/009.50.3.601-617.

Ortiz, Eduardo, Prats, José Ignacio, \& Arolas, Gracia 2004. La persona completa. Aproximación desde la Antropología, la Psicología y la Biología, Valencia: Edicep. Textos Universitarios, n. 2.

Paunonen, Sampo V, y Jackson, Douglas N. 2000. “What Is Beyond the Big Five? Plenty!” Journal of Personality 68 (5), 821-835. doi:https://doi.org/10.1111/14676494.00117

Pittman, Joe F., Keiley, Margaret K., Kerpelman, Jennifer L., y Vaughn, Brian E. 2011. "Attachment, Identity, and Intimacy: Parallels Between Bowl- 
by's and Erikson's Paradigms." Journal of Family Theory \& Review 3 (1), 32-46. DOI:10.1111/j.1756-2589.2010.00079.x

Pérez, Andrés. 2008. "La crítica de Levinas a la Filosofía Trascendental Moderna." En Emmanuel Lévinas. La filosofía como ética, edited by A. Martos, .......... . PUV: Universidad de Valencia.

Polo, Leonardo. 1997. Ética. Hacia una versión moderna de temas clasicos (segunda). Madrid: AEDOS-Unión Editorial.

Polo, Leonardo. 1998. Antropologia transcendental. Tomo I. La persona humana. Pamplona: EUNSA.

Polo, Leonardo. 2007a. ¿Quien es el hombre? Un espíritu en el tiempo (sexta edic.). Madrid: RIALP.

Polo, Leonardo. 2007b. Persona y libertad. Pamplona: EUNSA.

Spaemann, R. 2000. Personas. Pamplona: EUNSA.

Taylor, Charles. 2006. Las fuentes del yo. La construcción de la identidad moderna. Barcelona: Paidos Iberica.

Taylor, G. 1985. Pride, Shame and Guilt: Emotions of self-assessment. New York: Oxford University Press.

Téllez Maqueo, D.E. 2013. “La noción de moralismo en Josef Ratzinger.” Open Insight 4 (6), 115-131.

Thurstone, L.L. 1934. "The vectors of mind." Psychological Review 41, 1-32.

Tomasello, Michael. 2007. "Shared intentionality.” Developmental Science 10 (1), 121-125.

Tomasello, Michael. 2014. A natural history of human thinking. London: Harvard University Press.

Walden, T.A., y Kim, G. 2005. "Infants' social looking toward mothers and strangers.” International Journal of Behavioral Development 29 (5), 356-360. DOI:10.1177/01650250500166824

Walden, T.A., y Ogan, T.A. 1988. "The development of social referencing." Child Dev. 59 (5), 1230-1240.

Whitehead, Alfred N. 1978. Process and reality. An essay of cosmology. New York: The free press.

Winnicott, D.W. 1986. Home is where we start from. Essays by a psychoanalist. London: Penguin books.

Wojtyla, K. 1997. Mi visión del hombre. Madrid: Palabra.

Wojtyla, K. 1982. Persona y acción. Madrid: Ed. Biblioteca de Autores Cristianos. 